\title{
Superficial Peroneal Nerve Entrapment Causing Chronic Pain in the Foot: A Case Report
}

\author{
Jaehwan Kim¹, Chang-ik Lee ${ }^{1}$, Byung-chul Son ${ }^{1,2}$ \\ ${ }^{1}$ Department of Neurosurgery, Seoul St. Mary's Hospital, College of Medicine, The Catholic University of Korea, Seoul, Republic of Korea \\ ${ }^{2}$ Catholic Neuroscience Institute, College of Medicine, The Catholic University of Korea, Seoul, Republic of Korea
}

Corresponding author:

Byung-chul Son

Department of Neurosurgery, Seoul St. Mary's Hospital, Catholic Neuroscience Institute, College of Medicine, The Catholic University of Korea, 222 Banpo-daero, Seocho-gu, Seoul

06591, Republic of Korea

Tel: $+82-2-2258-6122$

Fax: +82-2-594-4248

E-mail: sbc@catholic.ac.kr

Received: September 23, 2021

Revised: September 28, 2021

Accepted: October 6, 2021

\begin{abstract}
The superficial peroneal nerve (SPN) supplies sensory innervation to the lower two-thirds of the lateral leg and the dorsum of the foot, with the exception of the first web space. Therefore, isolated entrapment of the SPN results in pain and sensory deficit of the dorsum of the foot or lateral lower leg without corresponding weakness of the peroneus muscle. Although entrapment of the SPN passing from the subfacial to the subcutaneous tissue in the peroneal tunnel was discovered as early as 1954, there were few reports of treatment of SPN entrapment. We report a case of a 73-year-old patient who presented with chronic numbness and allodynia of a 2-year duration due to entrapment of the SPN. Characteristic pain distribution and presentation of the symptoms were essential in preoperative diagnosis of SPN entrapment. The SPN and its branches were addressed in the peroneal tunnel and their courses were completely decompressed during the operation. Two months after the surgery, tingling pain and allodynia were recovered completely.
\end{abstract}

Key Words: Nerve compression syndromes; Pain; Peroneal nerve; Peroneal neuropathies

\section{INTRODUCTION}

While common peroneal nerve entrapment is a well-recognized pathology, symptomatic entrapment of the superficial peroneal nerve $(\mathrm{SPN})$ is rare and a more elusive clinical entity with a less established treatment plan ${ }^{4)}$. SPN entrapment was first described by Henry ${ }^{6}$ who observed that the pain could be reproduced by compressing the nerve at the point where it emerged from the deep fascia. In 1960, Kopell and Thompson ${ }^{7}$ suggested the site of entrapment where the SPN pieces the deep fascia in the distal leg. Styf and Morberg ${ }^{12}$ demonstrated the rarity of this entrapment syndrome in 1997. The incidence of SPN entrapment was 3.5\% of 480 patients with chronic leg pain. In addition, they reported an $80 \%$ success rate with decompression of the SPN ${ }^{12}$. Successful results of SPN decompression have been repeatedly reported since the report of Styf and Morberg.

The diagnosis and treatment of SPN compression have not been as clearly defined. Sensory disturbance of the dorsum of the foot or lateral lower leg without corresponding weakness of the peroneus muscles is hallmark of the entrapment of the superior peroneal nerve ${ }^{1)}$. Diagnosis is made mostly based on the clinical symptoms because it is difficult to diagnose with nerve conduction studies ${ }^{4,8,12)}$. Because there are multiple potential sites of peroneal nerve entrapment, it is difficult to ascertain whether potential compression sites are involved or whether the nerve is simply aggravated by the more proximal compression ". Entrapment of the superficial and common peroneal nerves may be concurrent. Franco et al." reported that $78 \%$ of patients with decompression of the SPN had been treated previously by common peroneal nerve decompression surgery. We report a patient who presented with chronic neuropathic pain in the left lower leg and foot, was diagnosed with isolated SPN entrapment, and was successfully treated with decompression surgery.

\section{CASE REPORT}

A 73-year-old male patient presented with burning and tingling pain in the left ankle and dorsum of the foot lasting 2 years. Two years ago, while he was squatting to work, a dull, slightly numb sensation suddenly developed in his left lower leg lateral to his shin (Fig. 1). This dull pain continued and was uncomfortable, but it did not interfere with the movement of the left leg and was not very severe. It lasted for 2 months and went away spontaneously without any special treatment. Six months later, while he squatted back to work, a stiff pain suddenly occurred in the previous painful area of his left lower leg. At the same time, burning pain and tingling sensation occurred in his ankle, dorsum of the foot, and toes (Fig. 1). The pain in his left lower leg and foot, which occurred again, did not improve naturally and lasted all day. The pain did not cause gait disturbance but got slightly worse as he walked. Over time, the stiffness in the left lower leg disappeared gradually, but the buming pain in his left ankle and the dorsum of left foot persisted.

Two years after the occurrence of pain, the pain in the left ankle and foot became more severe, which interfered with activities of daily life and work. He was diagnosed with lumbar spinal stenosis 

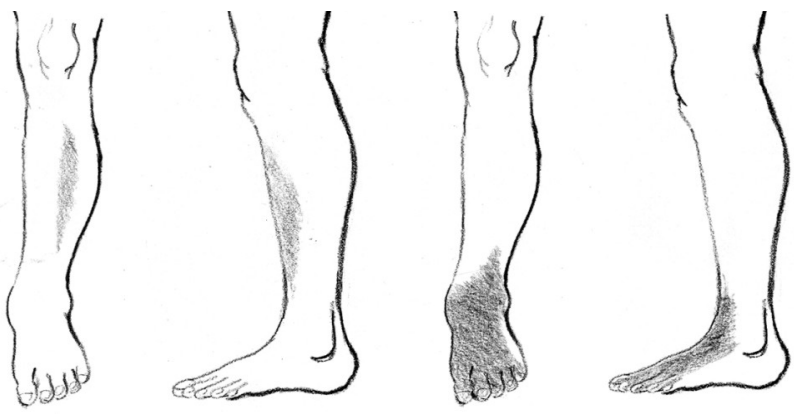

Fig. 1. Pain distribution of superficial peroneal entrapment. Drawing showing initial distribution of pain (left). The black area indicates the location of the stiff pain in his left lower leg that he developed while squatting 2 years ago. In the right side of the image, black area indicates the distribution of allodynic pain in the left ankle, the dorsum of the foot, and the toes.

at several orthopedic clinics and was treated with regular medications and epidural blocks without any improvement. Finally, electromyography (EMG) was recommended at a hospital for suspicion of peripheral nerve abnormalities. He was referred to the present author as abnormality in the conduction velocity of the SPN was confirmed by the examination.

On examination, there was no weakness of eversion of the left foot. Neither restriction of lumbosacral range of motion nor complaints of low back pain and ankle was observed. No tenderness or Tinel sign was evoked along the course of the peroneal nerve in the left lower leg. However, allodynia to light touch was elicited in his left ankle and dorsum of the foot. Mild hypesthesia was observed in the painful area. His pain was always present regardless of leg movement and posture and did not improve with rest. There was no pain induced by passive movement of the knee or ankle. However, he reported that the pain worsened after walking and momentarily became very severe when squatting. Sciatic root stretch signs were negative and the deep tendon reflex was normoactive.

An EMG and nerve conduction study of the lower extremity performed at one hospital showed an absence of discernible waves in the left SPN. However, no abnormality was found in the amplitudes of the tibialis anterior and peroneus muscles. Magnetic resonance imaging (MRI) of the left knee and ankle did not reveal any lesions that could explain the patient's pain. X-ray findings of the left knee, ankle, and foot were nonspecific. Laboratory examinations including enthrocyte sedimentation rate, C-reactive protein, rheumatoid factor, antinuclear antibody, and creatinine kinase were normal. Considering the course of neuralgia of the left SPN and abnormal EMG findings, an exploration of the left SPN was performed.

A $20 \mathrm{~cm}$-long, longitudinal skin incision was made two fingerbread ths lateral to the apex of the tibia, centering on the area where the initial pain occurred, $10 \mathrm{~cm}$ proximal to the lateral malleolus, as previously suggested (Fig. 2A) ${ }^{1,4)}$. The anterior intermuscular septum was easily identified after dissection of the subcutaneous layer (Fig. 2B). The SPN was identified under the fascial envelope lateral to the anterior intermuscular septum (Fig. 2B). The superficial fascia was divided longitudinally, revealing a $V$-shaped interfascial space

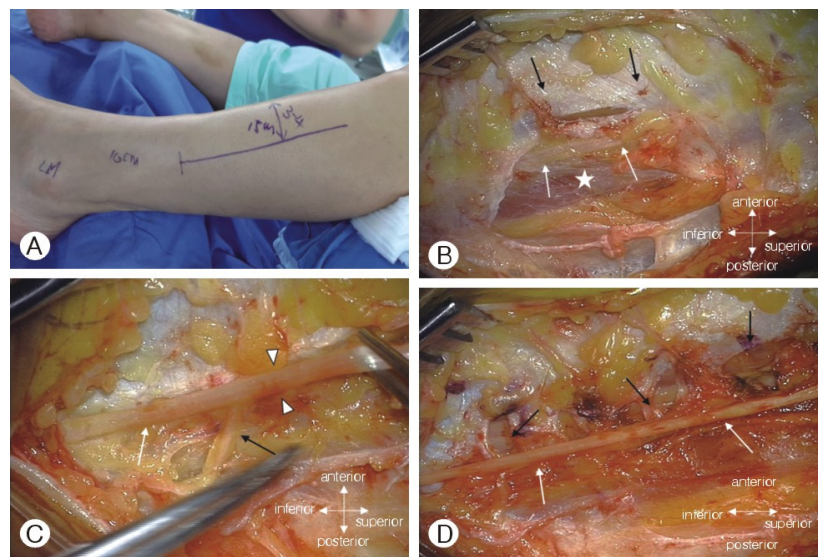

Fig. 2. Intraoperative findings during decompression of left superficial peroneal nerve. (A) A photograph showing the location of the skin incision marked on the anterolateral aspect of the distal leg along the expected path of the superficial peroneal nerve. (B) Initial exposure following dissection of the subcutaneous layer and the superficial peroneal nerve. The superficial peroneal nerve (white arrows) was identified in the lateral compartment after opening of the superficial fascia, lateral to the anterior compartment (black arrows). The peroneus longus muscle (white star) is visible just below the superficial peroneal nerve. (C) A photograph showing the branching of the superficial peroneal nerve in its distal peroneal tunnel. The medial dorsal cutaneous nerve of the foot (white arrow) and the intermediate dorsal cutaneous nerve of the foot (black arrow) are addressed. (D) A photograph showing complete dissection of the superficial peroneal nerve (white arrows). The anterior intermuscular septum (black arrows), which partitions the anterior and lateral compartments, was subjected to both longitudinal and transverse incisions so that the peroneal tunnel was sufficiently decompressed. LM: lateral malleolus.

within which the nerve run. In the distal side of the incision, the location of nerve entrapment was identified just prior to the bifurcating point into the intermediate and medial dorsal cutaneous sensory nerves (Fig. 2C). Proximal dissection along the course of SPN did not reveal any suspicious area of entrapment. After securing complete dissection of the proximal and distal course of the nerve, the fascial septum underlying the nerve was divided with four cuts to completely loosen the fascial tunnel (Fig. 2D). There were no anatomical variations, such as the passage of the SPN within the anterior compartment ${ }^{3)}$.

The allodynia in the left leg temporarily worsened for 2 weeks after the operation and then gradually improved until a month. The patient's condition was stable without any recurrent symptoms at the 12-month follow-up at an outpatient clinic.

\section{DISCUSSION}

\section{Anatomy of the SPN and Its Entrapment}

The SPN (superficial fibular nerve) begins at the bifurcation of the common peroneal nerve. It lies deep to the peroneus longus at first, then passed anterio-inferiorly between the peroneus longus 
and brevis muscles and extensor digitorum longus, and pierces the deep fascia in the distal third of the leg ${ }^{11)}$. It divides onto a large medial dorsal cutaneous nerve and a smaller, laterally placed, intermediate dorsal cutaneous nerve, usually after piercing the deep fascia, but sometimes while it is still deep to the fascia ${ }^{111}$. As the nerve lies between the muscles, it supplies the peroneus longus and brevis muscles and the skin of the lower leg.

The medial dorsal cutaneous nerve passes in front of the ankle joint and divides into two dorsal digital branches; one supplies the medial side of the hallux and the other supplies the adjacent side of the second and third toes ${ }^{10,11}$. It communicates with the saphenous and deep peroneal nerves. The smaller intermediate branch crosses the dorsum of the foot laterally. It divides into dorsal digital branches that supply the contiguous sides of the third to fifth toes and the skin of the lateral aspect of the ankle, where it connects with the sural nerve. Therefore, branches of the SPN supply the skin of the dorsum of all the toes except that of the lateral sided of the fifth toe (supplied by the sural nerve) and the adjoining sides of the great and second toes (supplied by the deep peroneal nerves).

A lesion of the SPN causes weakness of foot eversion and sensory loss on the lateral aspect of the leg that extends on to the dorsum of the foot. The nerve can be subjected to entrapment as it penetrates the deep fascia of the leg and it may also be involved in compartment syndrome that affects the lateral compartment of the $\mathrm{leg}^{10)}$. The path of the SPN varies significantly, traveling through the lateral compartment only, the anterior compartment, or both compartments ${ }^{3,10,11)}$. A cadaveric anatomical study revealed that the SPN branched before piercing the crural fascia in 35\% of the specimens, and in all these specimens the medial dorsal cutaneous nerve of the foot was located in the anterior compartment while the intermediate dorsal cutaneous nerve of the foot was located in the lateral compartment ${ }^{10}$. In addition, in 35\% of specimens, the intermediate dorsal cutaneous nerve of the foot was absent or did not innervate any toe. The sural nerve supplied the superficial innervation to the lateral half of the foot and toes in $40 \%$ of the specimens ${ }^{10)}$.

As it exits though the deep fascia at the distal third of the leg, where it divides into the medial and intermediate dorsal cutaneous nerve branches, the fascia area where the nerve pass though creates a site for entrapment ${ }^{3,5,10,11}$. This site of stenosis is further exacerbated by muscle hypertrophy, displaced fractures, direct trauma, mass effect, edema, and inversion injuries ${ }^{10)}$.

\section{Diagnosis}

Sensory disturbance of the dorsum of the foot or lateral lower leg without corresponding weakness of the peroneus muscles is hallmark of the entrapment of the superior peroneal nerve ${ }^{1)}$. The pain is aggravated by forceful inversion of the foot, which causes traction of the nerve against its entrapment site as it pierces the deep fascia of the distal leg. In addition, exacerbation of the symptoms by walking and exercise is common. Positive Tinel sign and transient improvement of the pain by local blockade of the SPN are diagnostically useful ${ }^{1,8)}$. The reported etiologies of SPN entrapment include muscle herniation, trauma, mass lesions such as lipoma and varicose vein, and idiopathic ${ }^{8}$.

Nerve conduction studies may be useful in demonstrating a local conduction defect along the course of the SPN in the absence of findings at the fibular head ${ }^{1,29}$. The normal function of the extensor digitorum brevis (EDB) is expected with preserved first web space sensation $^{1,2,9)}$. Except for the cases with mass lesions, the use of MRI, ultrasound, and other imaging modalities is not routinely performed ${ }^{4}$.

\section{Considerations in Surgical Treatment}

To prevent placing the SPN under tension, conservative treatment involves immobilization and limiting inversion and plantar flexion ${ }^{10)}$. A corticosteroid injection can be diagnostic and therapeutic. If conservative treatment fails, then surgical treatment involves the release of the entrapment site of the SPN ${ }^{10)}$.

The first thing to consider when performing decompression surgery is that the entrapment of the SPN may be multiple. As can be seen in the report of Franco et al. ${ }^{4}$, $78 \%$ of their patients who underwent SPN decompression surgery had combined simultaneous decompression of the common peroneal nerve. They hypothesized that an area proximal to the compression site might render areas further downstream more susceptible to compression (double crush phenomenon $)^{4,8)}$. Indeed, 5 patients reported in another case series also underwent common peroneal nerve decompression prior to SPN decompression ${ }^{8)}$. According to Franco et al. ${ }^{4)}$, those patients who had only decompression of the SPN without common peroneal release showed negative provocative maneuvers at the fibular head region, no motor weakness, and no electrophysiological evidence of motor disturbance. In the present case, no motor weakness was clinically confirmed, only sensory symptoms and pain of the SPN distribution, and no motor disturbance was identified in the EMG. In addition, as no mass lesion was found in the MRI, only decompression surgery of the SPN was performed.

The next consideration when performing decompression for the SPN entrapment is the possibility of anatomical variation of the nerve and complete decompression of its proximal and distal course $^{1,3,10)}$. An important anatomical variation to recognize is the accessory peroneal nerve from the SPN, which contributes innervation to the EDB in approximately one-third of the population ${ }^{2,9)}$. Long incisions in the front of the lower leg (up to $18 \mathrm{~cm}$ ), as suggested by experienced authors"), may be a little burdensome. However, a long incision along the course of the SPN seems inevitable to ensure complete decompression of the nerve penetration site of the deep fascia and to check the anatomical variations and possible communication branches leading to the anterior compartment ${ }^{1,3,10)}$. For example, in Matsumoto et al.'s report ${ }^{8)}$, a small incision in the first operation for SPN entrapment focused on the fascial entrapment site caused an insufficient nerve resulted in recurrence of the symptoms requiring additional decompression. A fasciotomy for the anterior and lateral compartment was recommended for complete loosening of the fascial entrapment site of the SPN and the fascial tunnel ${ }^{1,4)}$. Some authors recommend decompression of all three sites of possible entrapment of the superficial peroneal and common peroneal nerve pathways for successful pain relief ${ }^{1)}$. 


\section{CONCLUSION}

The entrapment of the SPN is a rare cause of leg and foot pain. Symptoms of SPN entrapment are of paramount importance for diagnosis. Understanding the anatomical variation of the nerve and complete decompression are thought to be important for successful treatment.

\section{CONFLICTS OF INTEREST}

No potential conflict of interest relevant to this article was reported.

\section{REFERENCES}

1. Boyd KU, Brown JM. Injury and compression neuropathy in the lower extremity. In: Mackinnon SE, Yee A eds. Nerve surgery. New York, NY: Thieme, 2015, pp338-390

2. Craig A: Entrapment neuropathy of the lower extremity. PM R 5:S31-S40, 2013

3. Ducic I, Dellon AL, Graw KS: The clinical importance of variations in the surgical anatomy of the superficial peroneal nerve in the mid-third of the lateral leg. Ann Plast Surg 56:635-638, 2006

4. Franco MJ, Phillips BZ, Lalchandani GR, Mackinnon SE: De- compression of the superficial peroneal nerve: clinical outcomes and anatomical study. J Neurosurg 126:330-335, 2017

5. Grear BJ. Neurogenic disorders. In: Azar FM, Beaty JH eds. Campbell's operative orthopaedics, ed 14th. Philadelphia, PA: Elsevier, 2021, pp4345-4381

6. Henry AK: Extensile exposure. Edinburgh, UK: E \& S Churchill Livingstone, 1945

7. Kopell HP, Thompson AL: Peripheral entrapment neuropathy of the lower extremity. N Engl J Med 262:56-60, 1960

8. Matsumoto J, Isu T, Kim K, Iwamoto N, Yamazaki K, Isobe $\mathrm{M}$ : Clinical features and surgical treatment of superficial peroneal nerve entrapment neuropathy. Neurol Med Chir (Tokyo) 58:320-325, 2018

9. Preston DC, Shapiro BE. Peroneal nerve palsy. In: Preston DC, Shapiro BE eds. Electromyography and neuromuscular disorders, ed 2nd. Philadelphia, PA: Elsevier, 2005, pp343-354

10. Solomon LB, Ferris L, Tedman R, Henneberg M: Surgical anatomy of the sural and superficial fibular nerves with an emphasis on the approach to the lateral malleolus. J Anat 199:717723, 2001

11. Standring S: Gray's anatomy: The anatomical basis of clinical practice, ed 42nd. New York, NY: Elsevier, 2020

12. Styf J, Morberg P: The superficial peroneal tunnel syndrome. Results of treatment by decompression. J Bone Joint Surg Br 79:801-803, 1997 